\title{
Effect of Bradyrhizobium Broth on Growth of Root, Shoot and Nodule of Soybean
}

\author{
Manisha S. Lande*, K.V. Bawankule, R.D. Solanki and R.G. Aware \\ Department of Plant Pathology, College of Agriculture, Nagpur \\ Dr. PDKV Akola (MS), India \\ *Corresponding author
}

\section{A B S T R A C T}

\begin{tabular}{|c|c|}
\hline $\begin{array}{l}\text { K e y w o r d s } \\
\text { Bradyrhizobium, } \\
\text { Soybean, Root, } \\
\text { Shoot, Nodule }\end{array}$ & $\begin{array}{l}\text { A pot culture experiment was conducted in the year } 2012-2013 \text { to test the performance of } \\
\text { glycerol based inoculant of Bradyrhizobium on growth of soybean using variety JS-335, at } \\
\text { Plant Pathology Department, College of Agriculture, Nagpur. Experiment was laid out in } \\
\text { Completely Randomized Design (CRD) with eight treatments and three replications. } \\
\text { During experiment, root and shoot length as well as number of nodules were measured at }\end{array}$ \\
\hline Article Info & $\begin{array}{l}15,30 \text { and } 45 \text { days after inoculation. Among all treatments, maximum root and shoot } \\
\text { length was recorded in T2, i.e.. } 35 \mathrm{ml} \text { glycerol }+65 \mathrm{ml} \text { Bradvrhizobium broth }(23.8 \mathrm{~cm} \text { and }\end{array}$ \\
\hline $\begin{array}{l}\text { Accepted: } \\
12 \text { June } 2019 \\
\text { Available Online: } \\
10 \text { July } 2019\end{array}$ & $\begin{array}{l}17.93 \mathrm{~cm} / \text { plant) respectively. However, maximum nodulation was recorded in T3, i.e., } 35 \\
\mathrm{ml} \text { glycerol }+65 \text { Bradyrhizobium broth }(11.00) \text {. All the treatments showed a significant } \\
\text { difference over untreated plot. }\end{array}$ \\
\hline
\end{tabular}

\section{Introduction}

Soybean (Glycine $\max$ L. Merill), family Leguminaceae is unique crop, versatile nutritional attribute yielding in both oil and protein. It is also known as "Golden Bean". The speedy growth in soybean cultivation has placed India in world map of soybean. India ranks fifth in the world, in area and production after USA, Brazil, China and Argentina. India is considered as a secondary center of demonstration of soybean. Soybean ranks first among oil seed crops in the world. However, it has manifold importance in agriculture, medicinal and industrial sector. It has high nutritive value, contains $40 \%$ protein, $20 \%$ oil, $30 \%$ carbohydrate and $5 \%$ fiber. It also contains vitamin A, B, C, D, E and other essential amino acids. Soybean is an excellent food available at the most economic rate. It is recommended in diabetes, stomach, heart and kidney disease.

Recent day's Rhizobium biofertilizers are widely used by farmers to increase legume crop yield. Biofertilizer can not be totally replace conventional chemical fertilizers but for most of crop and soil conditions up to 20 per cent of nitrogen requirement can be met through biofertilizer, which can be the best 
supplement for chemical fertilizer. It may help to reduce the cost of chemical fertilizer and avoid the soil problems.

Rhizobium belongs to bacterial group and classical example of symbiotic nitrogen fixation. Among the different BNF processes, legume Rhizobium symbiosis is the most effective means of nitrogen addition to terrestrial ecosystem. The bacteria infect the legume root and form root nodule within which they reduce molecular nitrogen to ammonia which is reutilized by plant to produce valuable proteins, vitamins and other nitrogen containing compounds. The site of symbiosis is within the root nodules. It has been estimated that $40-250 \mathrm{Kg} \mathrm{N} / \mathrm{ha} /$ year is fixed. But the effectiveness of biofertilizer as a nutrient input in crop production depend on efficiency of microbial strains i.e., their shelf life in a carrier and soil and weather parameters during crop growth, Although peat, charcoal are suitable as a carrier of biofertilizer. There is scope for development of better carrier to improve effectiveness of biofertilizer agents for longer shelf life.

Liquid biofertilizer is increasingly available in the market as one of the alternative to powder based biofertilizer. The shelf life of liquid biofertilizer is two to four years. The application of liquid formulation in the field is also very simple and easy. Liquid formulation contains special cell protectant or substance that encourages formation of resting spores or cysts. It contains special nutrients that ensure longer shelf life, better survival on seed and soil and tolerance to adverse conditions, to protect the microorganism from harmful environmental factors as the target site (field) increasing persistence.

The powder carrier based biofertilizer are used since a long time. The traditional nitrogen fixing biofertilizer have suffered from problems of short shelf life, instability to ambient temperature and laborious large scale application. Whereas liquid inoculants could be produced with minimum labour, space and energy and also the quantity of inoculum required is less compared to carrier based formulations. It is easier for farmers to handle. Liquid biofertilizer formulation could be considered as one potential strategy for improving the shelf life of biofertilizer. Unlike solid carrier based biofertilizers, liquid formulations allow the manufacturer to include sufficient amount of nutrients, cell protectant and inducers responsible for cell/spore/cyst formation to ensure prolonged shelf life.

In present study Bradyrhizobium japonicum was selected as test organism. It is slow growing bacterium of family Rhizobiaceae. Inoculation of soybean seed with Bradyrhizobium can fix 60 to $80 \mathrm{~kg}$ N/ha and subsequently increase the nodulation, shoot length of plant, plant height, number of pods and seed yield over control. Solid carriers are difficult to process to consistent characteristics and may not appropriate using with planting equipment used on large scale of field operation (Singleton et al., 2002). Liquid inoculants formulation is one solution to the problems associated with processing of solid carriers. The use of broth culture amended with substances that promote cells survival in the package and after application for seed.

\section{Materials and Methods}

An experiment was conducted in Plant Pathology Laboratory, College of Agriculture, Nagpur during the year 2012-2013. The seed of soybean (JS- 335) was obtained from Central Research Station, Dr. Panjabrao Deshmukh Krishi Vidyapeeth, Akola. Whereas, carrier material taken for study viz. glycerol, gum arabica, EDTA, PVP and lignite which were procured in ready condition (120 mesh). 


\section{Pure culture}

Pure culture of Bradyrhizobium was collected from Plant Pathology Section, College of Agriculture, Nagpur. This culture was used as test culture in the present investigation. All glassware sterilized in hot air oven at $180^{\circ} \mathrm{C}$ for 1 hour. Culture media and distilled water was sterilized in autoclave at $15 \mathrm{lbs}$ pressure for 15 minutes.

\section{Preparation of yeast extract mannitol agar}

For maintenance and purification of bacterial culture YEMA media was used. YEMA broth was used for mass multiplication of bacteria.

The medium was prepared by using the following ingredients.

\begin{tabular}{|c|c|}
\hline $\mathrm{K}_{2} \mathrm{HPO}_{4}$ & $-0.5 \mathrm{~g}$ \\
\hline $\mathrm{MgSo}_{4}-7 \mathrm{H}_{2} \mathrm{O}$ & $0.2 \mathrm{~g}$ \\
\hline Nacl & $0.1 \mathrm{~g}$ \\
\hline Mannitol & $-10 \mathrm{~g}$ \\
\hline Agar-Agar & $-20 \mathrm{~g}$ \\
\hline Congo red & $-2.5 \mathrm{ml}$ \\
\hline
\end{tabular}

Growth, maintenance and preservation of Bradyrhizobium culture

Pure culture of Bradyrhizobium was maintained on YEMA slants (Graham and Parker, 1967). Sub culturing and checking for purity was done once in two months when stored at $4^{0} \mathrm{C}$ temperatures.

\section{Pot culture experiment}

The investigation was conducted in pot culture by following Completely Randomized Design (C.R.D.) to test the performance of glycerol based inoculant of Bradyrhizobium on growth of soybean using variety JS335. Soybeanseedwere treated with glycerol based Bradyrhizobium broth and Rhizobium liquid biofertilizer available in market. The treated seeds were sown in pots contains sterilized soil according to treatment details and observations on growth parameters of soybean such as root length, shoot length and root nodule germination percent was recorded.

\section{For field work}

Soybean seed @ $1 \mathrm{Kg}$ was be treated as under

\begin{tabular}{|c|c|}
\hline Treatments & Treatment details \\
\hline $\mathrm{T}_{1}$ & $\begin{array}{l}15 \mathrm{ml} \text { glycerol }+85 \mathrm{ml} \\
\text { Bradyrhizobium broth }\end{array}$ \\
\hline $\mathrm{T}_{2}$ & $\begin{array}{l}25 \mathrm{ml} \text { glycerol }+75 \mathrm{ml} \\
\text { Bradyrhizobium broth }\end{array}$ \\
\hline $\mathrm{T}_{3}$ & $\begin{array}{l}35 \mathrm{ml} \text { glycerol +65 } \mathrm{ml} \\
\text { Bradyrhizobium broth }\end{array}$ \\
\hline $\mathrm{T}_{4}$ & 25g Powder base inoculants \\
\hline $\mathrm{T}_{5}$ & $\begin{array}{l}\text { Rhizobium liquid } \\
\text { biofertilizer(Market product- } \\
\text { 1) }\end{array}$ \\
\hline $\mathrm{T}_{6}$ & $\begin{array}{l}\text { Rhizobium liquid } \\
\text { biofertilizer(Market product- } \\
\text { 2) }\end{array}$ \\
\hline $\mathrm{T}_{7}$ & Untreated control \\
\hline
\end{tabular}

\section{Experimental details}

$\begin{array}{ll}\text { Duration } & -2012-13 \\ \text { Design } & - \text { CRD } \\ \text { Treatments } & -7 \\ \text { Replications } & -3 \\ \text { Variety } & - \text { Soybean JS-335 }\end{array}$

\section{Observation}

1. Root length: Root length was recorded after 15, 30, 45 days.

2. Shoot length: Shoot length was recorded after 15, 30, 45 days.

3. Nodulation: Plants from each pot were dug out carefully without loss of nodule along with soil in the field. The plants were then 
washed under tap water to remove soil adhering to root surface. The nodules were cut from the root carefully and their total number was recorded.

\section{Towel paper method}

Seed of soybean JS-335 was treated with different liquid inoculants at different concentration as per treatment details. Root length $(\mathrm{cm})$, shoot length $(\mathrm{cm})$, seedling vigour index and germination percentage were recorded by towel paper method.

The seedling vigour index was computed by using the following formula.

Vigour Index $=$ germination $(\%) \times[\operatorname{root}$ length $(\mathrm{cm})+$ shoot length $(\mathrm{cm})]$

The per cent seed germination was computed, based on number of seed germinated in a moistened paper towel where the hundred treated seed were placed and incubated at room temperature.

\section{Analysis of data}

The data of various experiments was analyzed using Complete Randomized Design. Analysis of variance, means were tested for significance and critical difference was used for comparison whenever the differences were found to the significant as indicated ' $F$ ' test (Gomez and Gomez, 1984)

\section{Results and Discussion}

Studies were undertaken on "Performance of Glycerol Based Inoculant of Bradyrhizobium Growth on Soybean" during the year 2012 in Completely Randomized Design (CRD) with eight treatments and three replications using the soybean variety JS-335. The results are presented in tables, photograph and figures are depicted in each head in this chapter.

\section{Rhizobial population in glycerol carrier}

Two substrates viz., glycerol and lignite powder mixed individually with broth of Bradyrhizobium japonicum at $10^{7}$ cells $/ \mathrm{ml}$. The experiment was set for 180 days. The data obtained is presented in Table 1.

It was revealed from the data that there were significant differences in rhizobial population at all the intervals. Maximum population was attained with $10 \mathrm{ml}$ glycerol (61.66 X $10^{7}$ cell $/ \mathrm{ml}$.) and it was found significantly superior over all other treatment at 120 days in respect to other treatment next to $10 \mathrm{ml}$ glycerol treatment maximum rhizobial population was noticed in $85 \mathrm{ml}$ Bradyrhizobium broth $+15 \mathrm{ml}$ glycerol (56.66 $\mathrm{X} 10^{7}$ cells $/ \mathrm{ml}$.) and found significantly superior over other treatments. And it was followed by $\mathrm{T}_{3}(80 \mathrm{ml}$ Bradyrhizobium broth $+20 \mathrm{ml}$ glycerol), $\mathrm{T}_{5}$ (70 $\mathrm{ml}$ Bradyrhizobium broth $+30 \mathrm{ml}$ glycerol $), \mathrm{T}_{4} \quad(75 \mathrm{ml}$ Bradyrhizobium broth $+25 \mathrm{ml}$ glycerol), $\mathrm{T}_{6}$ $(65 \mathrm{ml}$ Bradyrhizobium broth $+35 \mathrm{ml}$ glycerol) showed the rhizobial population $35 \mathrm{x}$ $10^{7} \mathrm{cell} / \mathrm{ml}, 33.66 \times 10^{7} \mathrm{cell} / \mathrm{ml}, 32.66 \times 10^{7}$ cell $/ \mathrm{ml}$ and $31.33 \times 10^{7}$ cell $/ \mathrm{ml}$ after 120 days, respectively. There is decrease in rhizobial population was observed after 120 days. But in treatment $\mathrm{T}_{7}(25 \mathrm{~g}$ lignite $+75 \mathrm{ml}$ broth $)$ and $\mathrm{T}_{8}$ (Control) noticed high rhizobial population after 60 days i.e. $27.00 \times 10^{7}$ cell $/ \mathrm{ml}, 25.33 \times 10^{7}$ cell $/ \mathrm{ml}$ respectively. This may be due to the more water holding of carrier material. These results are in accordance with earlier studies of scientist Lorda and Balatti (1996) showed that the more rapid growth in a balanced medium that used $10 \mathrm{ml}$ glycerol as a substitute for mannitol. Sridhar et al. (2004) stated that higher population up to storage period of 180 days. Brahmaprakash (2011) showed lignite recorded maximum rhizobial colonies $(27 \mathrm{X}$ $10^{6}$ cells $\left./ \mathrm{ml}\right)$ at 60 days. Kandasamy and Prasad (1971) also reported that lignite was 
better carrier material for rhizobial population.

\section{Effect of carrier on the root length}

The data in respect of root length are given in Table 2 plant root length were recorded at 15 , 30,45 DAS. It is seen from the data that root length was significantly affected by various treatments over control. The maximum root length $(23.80 \mathrm{~cm})$ was recorded after 45 days by $25 \mathrm{ml}$ glycerol $+75 \mathrm{ml}$ Bradyrhizobium broth treatment $\left(\mathrm{T}_{2}\right)$. Followed by $\left(\mathrm{T}_{3}\right)$ treatment, $35 \mathrm{ml}$ glycerol $+65 \mathrm{ml}$ Bradyrhizobium broth $(23.63 \mathrm{~cm})$ root length was recorded and it was found to be significantly superior over all other treatment. Followed by treatment, $\mathrm{T}_{4}(25 \mathrm{~g}$ powder base inoculants), i.e. $21.00 \mathrm{~cm}$ and both the treatment $\mathrm{T}_{1}(85 \mathrm{ml}$ Bradyrhizobium broth + $15 \mathrm{ml}$ glycerol) and $\mathrm{T}_{5}$ (Rhizobium liquid biofertilizer (Market product-2) showed the same root length i.e. $20.16 \mathrm{~cm}$ at 45 DAS. The minimum root length was observed in treatment $\mathrm{T}_{6}$ (Rhizobium liquid biofertilizer (Market product-2) followed by $\mathrm{T}_{7}$ (Untreated) i.e., 19.96 and $15.78 \mathrm{~cm}$ respectively

\section{Effect of carrier on the shoot length $(\mathrm{cm})$}

As regards to shoot length, the data presented in Table 3 showed significant differences over control. The maximum shoot length was recorded in treatment $\mathrm{T}_{2}(25 \mathrm{ml}$ glycerol +75 $\mathrm{ml}$ Bradyrhizobium broth) i.e., 18.16 $\mathrm{cm} /$ plant) as compared to all other treatment. Next best treatment $\mathrm{T}_{3}(35 \mathrm{ml}$ glycerol +65 $\mathrm{ml}$ Bradyrhizobium broth), i.e. $17.93 \mathrm{~cm} /$ plant over all other remaining treatments. And further followed by $\mathrm{T}_{5}$ (Rhizobium liquid biofertilizer (Market product-1), $\mathrm{T}_{1}(15 \mathrm{ml}$ glycerol $+85 \mathrm{ml}$ Bradyrhizobium broth), $\mathrm{T}_{4}$ (25g powder base inoculants), $\mathrm{T}_{6}$ (Rhizobium liquid biofertilizer (Market Product-2) was recorded $17.86 \mathrm{~cm}, 17.36 \mathrm{~cm}, 16.86 \mathrm{~cm}$,
$15.96 \mathrm{~cm}$ respectively, after 45 DAS. All the treatment increased the shoot length over control. Treatment $\mathrm{T}_{8}$ (Control) was recorded minimum shoot length of plant $15.46 \mathrm{~cm} /$ plant.

\section{Effect of carrier on number of nodules per plant}

A pot culture experiment was conducted to test the efficacy of the carrier materials on nodulation of soybean crop and the result are tabulated in Table 4. The Maximum nodulation was noticed in $35 \mathrm{ml}$ glycerol +65 $\mathrm{ml}$ Bradyrhizobium broth (11.00 nodules/ plant) and it was significantly superior over all other treatment. The next treatment $\mathrm{T}_{2}$ (75 $\mathrm{ml}$ Bradyrhizobium broth $+25 \mathrm{ml}$ glycerol) was found significantly better over other treatment recorded 10.66 nodules/plant. Followed by $\mathrm{T}_{4}(25 \mathrm{~g}$ powder base inoculants), $\mathrm{T}_{5}$ (Rhizobium liquid biofertilizer Market product -1), $\mathrm{T}_{6}$ (Rhizobium liquid biofertilizer (Market product -2), $\mathrm{T}_{1}(85 \mathrm{ml}$ Bradyrhizobium broth $+15 \mathrm{ml}$ glycerol), recorded no. of nodules per plant i.e. 10.36 nodules/plant, 9.66 nodules/plant, 8.33 nodules/plant and 6.33 nodules/plant after 45 days. Seed inoculation with Rhizobium increased the nodulation. These findings are in agreement with the reports Kurundkar et al. (1991) and Chore and Shastri (1991). Benifical effect of carrier on nodulation was earlier reported by Jadhav et al. (1988).

\section{Effect of various treatment on root and shoot length by paper towel method}

Seeds of soybean variety JS-335 were treated with glycerol and liquid based inoculants (Market products) as described in treatment and inoculated with Bradyrhizobium japonicum for recording shoot length, root length, germination and seedling vigour index by using paper towel method. There were significant differences noticed due to various 
treatment and data presented in Table 5 reveals that maximum root and shoot length was recorded by $\left(\mathrm{T}_{1}\right) 15 \mathrm{ml}$ glycerol $+85 \mathrm{ml}$ Bradyrhizobium broth $(11.23 \mathrm{~cm}$ and $(9.20$ $\mathrm{cm})$ followed by $\left(\mathrm{T}_{4}\right)$ powder base treatment noticed (10.33) root length, and (7.23) shoot length. Further followed by $\mathrm{T}_{6}$ (Rhizobium liquid biofertilizer (Market product-2) with $9.66 \mathrm{~cm}$ root length $7.20 \mathrm{~cm}$, shoot length, $T_{3}$ $(35 \mathrm{ml}$ glycerol $+65 \mathrm{ml}$ Bradyrhizobium broth), with $9.43 \mathrm{~cm}$ root length $6.13 \mathrm{~cm}$ shoot length, $\mathrm{T}_{2}(25 \mathrm{ml}$ glycerol $+75 \mathrm{ml}$ Bradyrhizobium broth) with $9.30 \mathrm{~cm}$ root length and $6.30 \mathrm{~cm}$ shoot length. Minimum root length $\mathrm{T}_{7}$ (Control) recorded the root length $9.23 \mathrm{~cm}$, shoot length $5.8 \mathrm{~cm}$, and $\mathrm{T}_{6}$ (Rhizobium liquid biofertilizer (Market product-2) was recorded root length $8.20 \mathrm{~cm}$, and shoot length $6.53 \mathrm{~cm}$. Adu and Misari (1989) reported improvement of shoot length due to the inoculation of Rhizobium.
The data presented in above Table showed that treatment $\mathrm{T}_{2}(25 \mathrm{ml}$ glycerol $+75 \mathrm{ml}$ Bradyrhizobium broth) registered maximum germination percentage i.e. $79.3 \%$ which is significantly superior over all the other treatments. Next best treatment $\mathrm{T}_{1}(15 \mathrm{ml}$ glycerol $+85 \mathrm{ml}$ Bradyrhizobium broth) registered i.e. $77.3 \%$. And further followed by $\mathrm{T}_{4}$ (25 g powder base inoculant) i.e. $76.6 \%$, and treatment $\mathrm{T}_{3}(35 \mathrm{ml}$ glycerol $+65 \mathrm{ml}$ Bradyrhizobium broth) and $\mathrm{T}_{5}$ (Rhizobium liquid biofertilizer (Market product-1) showed the same germination percentage with $74 \%$. And the minimum germination percentage was recorded in treatment $\mathrm{T}_{6}$ (Rhizobium liquid biofertilizer (Market product-2) i.e. $74 \%$ followed by $\mathrm{T}_{7}$ (Control) with $72 \%$. Penaranda et al. (1988) observed the enhancement of germination due to Rhizobium inoculation on soybean, mungbean and pea.

Table.1 Rhizobial population (x $10^{7}$ cells $/ \mathrm{ml}$ of carrier) in glycerol carrier

\begin{tabular}{|c|c|c|c|c|c|c|c|}
\hline \multirow{2}{*}{$\begin{array}{l}\text { Tr. } \\
\text { No. }\end{array}$} & \multirow[t]{2}{*}{ Treatment } & \multicolumn{6}{|c|}{ Months } \\
\hline & & I & II & III & IV & $\mathbf{V}$ & VI \\
\hline $\mathbf{T}_{1}$ & $\begin{array}{l}\text { 90ml Bradyrhizobium broth }+10 \mathrm{ml} \\
\text { glycerol }\end{array}$ & 34.33 & 42.33 & 48.33 & 61.66 & 52.00 & 44.66 \\
\hline $\mathbf{T}_{2}$ & $85 \mathrm{ml}$ Bradyrhizobium broth $+15 \mathrm{ml}$ glycerol & 28.66 & 31.66 & 41.66 & 56.66 & 49.33 & 40.33 \\
\hline $\mathbf{T}_{3}$ & 80ml Bradyrhizobiumbroth+20ml glycerol & 24.66 & 26.33 & 30.33 & 35.00 & 32.33 & 29.66 \\
\hline $\mathbf{T}_{4}$ & $75 \mathrm{ml}$ Bradyrhizobium broth $+25 \mathrm{ml}$ glycerol & 19.66 & 28.00 & 31.66 & 32.66 & 30.66 & 26.00 \\
\hline $\mathbf{T}_{5}$ & 70ml Bradyrhizobium broth+30ml glycerol & 19.33 & 21.66 & 29.66 & 33.66 & 32.00 & 29.00 \\
\hline$T_{6}$ & 65ml Bradyrhizobium broth $+35 \mathrm{ml}$ glycerol & 18.33 & 26.33 & 29.00 & 31.33 & 28.00 & 24.66 \\
\hline $\mathbf{T}_{7}$ & $25 \mathrm{~g}$ lignite $+75 \mathrm{ml}$ Bradyrhizobium broth & 26.00 & 27.00 & 24.33 & 20.66 & 18.00 & 17.66 \\
\hline$T_{8}$ & Control & 22.33 & 25.33 & 20.33 & 19.66 & 17.66 & 17.33 \\
\hline & F test & Sig. & Sig. & Sig. & Sig. & Sig. & Sig. \\
\hline & $\mathrm{SE} \pm(\mathrm{m})$ & 1.39 & 1.25 & 1.49 & 1.38 & 1.48 & 1.58 \\
\hline & $\mathrm{CD}(\mathrm{P}=\mathbf{0 . 0 5})$ & 4.18 & 3.73 & 4.48 & 4.14 & 4.44 & 4.74 \\
\hline
\end{tabular}


Table.2 Effect of carrier on the root length $(\mathrm{cm})$

\begin{tabular}{|c|c|c|c|c|}
\hline Tr.No. & Treatment & 15 DAS & 30 DAS & 45 DAS \\
\hline $\mathbf{T}_{1}$ & $\begin{array}{l}85 \mathrm{ml} \text { Bradyrhizobium } \\
\text { broth }+15 \mathrm{ml} \text { glycerol }\end{array}$ & 5.20 & 11.70 & 20.16 \\
\hline $\mathbf{T}_{2}$ & $\begin{array}{l}25 \mathrm{ml} \text { glycerol }+75 \mathrm{ml} \\
\text { Bradyrhizobium broth }\end{array}$ & 7.90 & 16.06 & 23.80 \\
\hline $\mathbf{T}_{\mathbf{3}}$ & $\begin{array}{l}35 \mathrm{ml} \text { glycerol }+65 \mathrm{ml} \\
\text { Bradyrhizobium broth }\end{array}$ & 7.83 & 15.16 & 23.63 \\
\hline $\mathbf{T}_{4}$ & $\begin{array}{l}25 \mathrm{~g} \text { powder base } \\
\text { inoculants }\end{array}$ & 5.06 & 11.16 & 21.00 \\
\hline $\mathbf{T}_{5}$ & $\begin{array}{lr}\text { Rhizobium } & \text { liquid } \\
\text { biofertilizer } & \text { (Market } \\
\text { product-1) } & \end{array}$ & 5.26 & 13.80 & 20.16 \\
\hline $\mathbf{T}_{6}$ & $\begin{array}{lr}\text { Rhizobium } & \text { liquid } \\
\text { biofertilzer } & \text { (Market } \\
\text { product-2) } & \\
\end{array}$ & 5.16 & 11.50 & 19.96 \\
\hline \multirow[t]{4}{*}{$\mathbf{T}_{7}$} & Control & 5.03 & 8.16 & 15.78 \\
\hline & F test & Sig. & Sig. & Sig. \\
\hline & $\mathrm{SE} \pm(\mathrm{m})$ & 0.14 & 0.25 & 0.34 \\
\hline & $\mathrm{CD}(\mathrm{P}=0.05)$ & 0.52 & 0.77 & 1.08 \\
\hline
\end{tabular}

Table.3 Effect on carrier on the shoot length $(\mathrm{cm})$

\begin{tabular}{|c|c|c|c|c|}
\hline Tr.No. & Treatment & 15 DAS & 30 DAS & 45 DAS \\
\hline $\mathbf{T}_{1}$ & $\begin{array}{l}15 \mathrm{ml} \text { glycerol } \\
\text { Bradyrhizobium broth }\end{array}$ & 12.83 & 14.9 & 17.36 \\
\hline $\mathbf{T}_{2}$ & $\begin{array}{l}25 \mathrm{ml} \text { glycerol }+75 \mathrm{ml} \\
\text { Bradyrhizobium broth }\end{array}$ & 14.9 & 17.16 & 18.16 \\
\hline $\mathbf{T}_{\mathbf{3}}$ & $\begin{array}{l}35 \mathrm{ml} \text { glycerol }+65 \mathrm{ml} \\
\text { Bradyrhizobium broth }\end{array}$ & 14.1 & 15.43 & 17.93 \\
\hline $\mathbf{T}_{4}$ & $25 \mathrm{~g}$ powder base inoculants & 12.2 & 14.8 & 16.86 \\
\hline $\mathbf{T}_{\mathbf{5}}$ & $\begin{array}{l}\text { Rhizobium liquid biofertilizer } \\
\text { (Market product-1) }\end{array}$ & 13 & 15.2 & 17.86 \\
\hline $\mathbf{T}_{6}$ & $\begin{array}{l}\text { Rhizobium liquid biofertilzer } \\
\text { (Market product-2) }\end{array}$ & 12.53 & 12.13 & 15.96 \\
\hline \multirow[t]{4}{*}{$\mathbf{T}_{7}$} & Control & 11.70 & 13.76 & 15.46 \\
\hline & F test & Sig. & Sig. & Sig. \\
\hline & $\mathrm{SE} \pm(\mathrm{m})$ & 0.60 & 0.16 & 0.29 \\
\hline & $\mathrm{CD}(\mathrm{P}=0.05)$ & 0.19 & 0.51 & 0.89 \\
\hline
\end{tabular}


Table.4 Effect of carrier on number of nodules per plant

\begin{tabular}{|c|l|c|}
\hline \multicolumn{1}{|c|}{ Tr.No. } & \multicolumn{1}{|c|}{ Treatment } & No. of nodule/plant \\
\hline $\mathbf{T}_{\mathbf{1}}$ & $85 \mathrm{ml}$ Bradyrhizobium broth $+15 \mathrm{ml}$ glycerol & $\mathbf{6 . 3 3}$ \\
\hline $\mathbf{T}_{\mathbf{2}}$ & $75 \mathrm{ml}$ Bradyrhizobium broth $+25 \mathrm{ml}$ glycerol & $\mathbf{1 0 . 6 6}$ \\
\hline $\mathbf{T}_{\mathbf{3}}$ & $65 \mathrm{ml}$ Bradyrhizobium broth $+35 \mathrm{ml}$ glycerol & $\mathbf{1 1 . 0 0}$ \\
\hline $\mathbf{T}_{\mathbf{4}}$ & $25 \mathrm{~g}$ powder base inoculants & $\mathbf{1 0 . 3 6}$ \\
\hline $\mathbf{T}_{\mathbf{5}}$ & Rhizobium liquid biofertilizer (Market product-1) & $\mathbf{9 . 6 6}$ \\
\hline $\mathbf{T}_{\mathbf{6}}$ & Rhizobium liquid biofertilizer (Market product-2) & $\mathbf{8 . 3 3}$ \\
\hline $\mathbf{T}_{\mathbf{7}}$ & Control & $\mathbf{0 . 0 0}$ \\
\hline & F test & $\mathbf{S i g}$. \\
\hline & SE $\pm(\mathrm{m})$ & $\mathbf{0 . 6 7}$ \\
\hline & $\mathbf{C D}(\mathbf{P}=\mathbf{0 . 0 5})$ & $\mathbf{2 . 0 5}$ \\
\hline
\end{tabular}

Table.5 Effect of various treatment on root and shoot length by paper towel method

\begin{tabular}{|c|c|c|c|c|c|}
\hline Tr.No. & Treatment & $\begin{array}{c}\text { Root } \\
\text { length } \\
(\mathrm{cm} / \mathrm{pl} .)\end{array}$ & $\begin{array}{l}\text { Shoot } \\
\text { length } \\
(\mathrm{cm} / \mathrm{pl} .)\end{array}$ & Germination & SVI \\
\hline $\mathbf{T}_{1}$ & $\begin{array}{l}15 \mathrm{ml} \text { glycerol+85ml } \\
\text { Bradyrhizobium broth }\end{array}$ & 11.23 & 9.20 & $77.3 \%$ & 1579 \\
\hline $\mathbf{T}_{2}$ & $\begin{array}{l}25 \mathrm{ml} \text { glycerol+75ml } \\
\text { Bradyrhizobium broth }\end{array}$ & 9.30 & 6.30 & $79.3 \%$ & 1237 \\
\hline $\mathbf{T}_{\mathbf{3}}$ & $\begin{array}{l}35 \mathrm{ml} \text { glycerol+65 } \mathrm{ml} \\
\text { Bradyrhizobium broth }\end{array}$ & 9.43 & 6.13 & $75 \%$ & 1167 \\
\hline $\mathbf{T}_{4}$ & $25 \mathrm{~g}$ powder base inoculants & 10.33 & 7.23 & $76.6 \%$ & 1344 \\
\hline $\mathbf{T}_{5}$ & $\begin{array}{lr}\text { Rhizobium } & \text { liquid } \\
\text { biofertilizer } & \text { (Market } \\
\text { product-1) } & \end{array}$ & 8.20 & 6.53 & $75 \%$ & 1104 \\
\hline $\mathbf{T}_{6}$ & $\begin{array}{l}\text { Rhizobium } \\
\text { biofertilizer } \\
\text { product-2) }\end{array}$ & 9.66 & 7.20 & $74 \%$ & 1247 \\
\hline $\mathbf{T}_{7}$ & Control & 9.23 & 5.8 & $72 \%$ & 1082 \\
\hline & F test & Sig. & Sig. & Sig & - \\
\hline & $\mathrm{SE} \pm(\mathrm{m})$ & 0.1 & 0.10 & & - \\
\hline & $\mathrm{CD}(\mathrm{P}=\mathbf{0 . 0 5})$ & 0.30 & 0.34 & - & - \\
\hline
\end{tabular}

The results recorded in the above Table reveals that the seedling vigour index of soybean increased significantly in treatment
$\mathrm{T}_{1}$ (15 ml glycerol $+85 \mathrm{ml}$ Bradyrhizobium broth) i.e. increased by 1579 over uninoculated treatment. The remaining 
treatments i.e. $\mathrm{T}_{4} \quad(25 \mathrm{~g}$ powder base inocualant), $\mathrm{T}_{6}$ (Rhizobium liquid biofertilizer (Market product-2), $\mathrm{T}_{2}$ (25 $\mathrm{ml}$ glycerol +75 $\mathrm{ml}$ Bradyrhizobium broth), $\mathrm{T}_{3}$ (35 $\mathrm{ml}$ glycerol $+65 \mathrm{ml}$ Bradyrhizobium broth), $\mathrm{T}_{5}$ (Rhizobium liquid biofertilizer (Market product-1) which gave 1344, 1247, 1237, 1167 and 1104 respectively. The minimum seedling vigour index was recorded in uninoculated control i.e., 1082.

\section{References}

Lorda and Balatti. 1996. Selection and characterization of strain, production, use and management. Edi. Kingraf, Buenos Aires: 148.

Sridhar, V., G. P. Brahmaprakash, and S. V. Hegde. 2004. Development of liquid Inoculant using for phosphate solubilizing bacterium. Karnataka J. Agric. Sci.17 (2): 251-257.

Brahamprakash, G. P. and S. Velineni. 2011. Survival and phosphate solubilizing ability of Bacillus megaterium in liquid inoculants under high temperature and desiccation stress. Department of Agri.
Microbiology. 14 (5): 795-802.

Kandasami, R. and N. N. Prasad, 1971.Lignite as a carrier of Rhizobia.Curr. Sci. 40: 496.

Kurundkar, B. P., P. A. Thombre and B. R. Kawale. 1991. Effect of different inoculums levels of Bradyrhizobium japonicum on soybean. Legume Res. 14 (4): 201-204.

Chore, C. N. and N. R. Shastri. 1991. Study of soybean (Glycine max L. Merrill) to Rhizobium culture. J. Soil and Crops. 1 (2): 162-164.

Jadhav, V. T., S. S. Kore and C. D. Mayee. 1988. Influence of carriers, isolates and inoculation method of Rhizobium on groundnut in vertisols. J. Oliseed. Res. 5(2): 89-92.

Penaranda, F. L., H. L. Mendoza, G. R. Sahlao and T. Bod, 1988.Screening of different strains of Rhizobium on soybean, mungbean and peanut Phillippine J. Crop Sci. 13: 33-37.

Adu, J. K. and S. M. Misari. 1989. The influence of Rhizobium inoculation on the performance of groundnut (Arachis hypogea). Savana.10 (1): 11-17.

\section{How to cite this article:}

Manisha S. Lande, K.V. Bawankule, R.D. Solanki and Aware, R.G. 2019. Effect of Bradyrhizobium Broth on Growth of Root, Shoot and Nodule of Soybean. Int.J.Curr.Microbiol.App.Sci. 8(07): 1588-1596. doi: https://doi.org/10.20546/ijcmas.2019.807.189 